“ (C) 2017 IEEE. Personal use of this material is permitted. Permission from IEEE must be obtained for all other uses, in any current or future media, including

reprinting/republishing this material for advertising or promotional purposes, creating new collective works, for resale or redistribution to servers or lists, or reuse of any copyrighted component of this work in other works." 


\title{
A New Robust Beamforming Algorithm: Embedding Array's Active Pattern in Diagonal Loading Method
}

\author{
Yuyue Luo ${ }^{* * 1}$, Jin Pan ${ }^{\# 2}$, Shaode Huang ${ }^{\# 3}$ \\ \# School of Electronic Engineering, University of Electronic Science and Technology of China \\ * Faculty of Engineering and Information Technology, University of Technology Sydney \\ ${ }^{1}$ yyl uestc@163.com \\ 2panjin@uestc.edu.cn \\ ${ }^{3}$ ShaodeHuang@std.uestc.edu.cn
}

\begin{abstract}
In this paper, we propose a novel adaptive beamforming algorithm combining methods of electromagnetism and signal processing, which embeds antennas' active pattern (AP) in the diagonal loading (DL) method. It can significantly reduce the beamformer's performance degradation brought by the inconsistency between hypothesized idealized antenna models and practical ones. Embedding AP adds the array's electromagnetic characteristics to the algorithm, therefore improves the performance of the robust beamformer. Simulation and measurement results indicate that the proposed method has an appealing performance when there are mismatches of antennas' physical aperture.
\end{abstract}

Keywords - adaptive beamforming; array manifold mismatches; robust beamforming; active pattern; diagonal loading

\section{INTRODUCTION}

Widely used in in many fields such as sonar, radar, communications and navigation, the adaptive beamforming system performs well in selecting useful information, antiinterference and reducing noise. In traditional theory, antennas are assumed to be isotropic, identical, and omnidirectional elements placed in infinite free space. However, the ideal assumption can cause serious performance degradations in practical implementations due to the ignored array's imperfections (e.g., gain and phase mismatches, and mutual coupling between elements), particularly for increasingly widely used miniature apertures. Robust beamforming algorithms have been proposed to deal with these imperfections by treating array's response inconsistencies as non-specific manifold mismatches. One of the most popular robust beamforming approaches is the diagonal loading (DL) method (also known as loaded sample matrix inversion (LSMI) beamformer) [1], whose main idea is to add a quadratic penalty variable to the objective function and regularize the solution for the weight vector. As [1] does not give a clear way to obtain the value of DL factor, the worst-case-based [2] and other methods [3-5] are proposed to improve it. According to [3-5], most robust beamforming methods solve uncertain problems based on mathematical modelling and analysis of the signal (e.g., statistics, optimization, and eigenanalysis of matrix), but do not particularly analyze the array's electromagnetic characteristics. [6] reports some work exploiting the gain and frequency properties of practical antennas, without considering mutual coupling effect. [7] improves by incorporating the antenna's active pattern (AP) [8], which has a distinctive ability of calculating elements' radiation including its impact on the array environment (both mutual coupling between elements and workspace radiation). However, it greatly relies on the exact information of antennas' electromagnetic characteristics and is quite sensitive to measurement mismatches.

In this paper, we creatively propose the active pattern based diagonal loading (APDL) method by employing the AP method in the DL algorithm. Via both simulation and experiments with real measurements, we demonstrate that the APDL beamformer achieves an appealing performance (e.g., higher signal to interference and noise ratio, SINR). It has better tolerance to both engineering and electromagnetic mismatches caused by elements' modeling, manufacturing, aperture assembling, and channel debugging.

\section{PROBLEM Formulation}

A narrowband system with an M-element two-dimension antenna array is considered. Without considering any imperfections, its steering vector $\boldsymbol{a}$ can be written as:

$$
\boldsymbol{a}(f, \theta, \varphi, \boldsymbol{r})=\left[e^{j k k_{1}}, \cdots, e^{j k r_{M}}\right]^{T}
$$

where $k=2 \pi f / v$ is the wavenumber, $f$ and $v$ denote frequency and the speed of the electromagnetic wave, respectively. $\theta$ and $\varphi$ are the angles, and $\boldsymbol{r}_{i}$ is the location vector of the $i$ th sensor.

Assume antenna elements are omnidirectional. Let $s(t)$ and $i(t)$ be the transmitted data symbol and interference symbol respectively, at time $t$. The signal received at the array can be given by:

$$
\boldsymbol{x}(t)=\boldsymbol{s}(t)+\boldsymbol{i}(t)+\boldsymbol{n}(t)=s(t) \boldsymbol{a}_{s}+i(t) \boldsymbol{a}_{i}+\boldsymbol{n}(t)
$$

where $\boldsymbol{x}(t)=\left[x_{1}(t), x_{2}(t) \cdots, x_{M}(t)\right]^{T} \in C^{M \times 1}$ is the complex vector of array observations. $\boldsymbol{a}_{s}$ and $\boldsymbol{a}_{i}$ are the steering vectors of signal and interference respectively, and $\boldsymbol{n}(t)$ is an $M \times 1$ vector denoting the combined interference and noise components.

The sample matrix inversion (SMI) beamformer is used as the foundation algorithm, which solves a constrained minimization problem:

$$
\min _{\boldsymbol{w}} \boldsymbol{w}^{H} \hat{\boldsymbol{R}} \boldsymbol{w} \quad \text { s.t. } \quad \boldsymbol{w}^{H} \boldsymbol{a}_{s}=1 .
$$

where $\boldsymbol{w}=\left[w_{1}, w_{2}, \cdots, w_{M}\right]^{T} \in C^{M \times 1}$ is beamformer's complex vector weights, and $\hat{\boldsymbol{R}}$ is the sample covariance matrix of 
signal. $(\cdot)^{\mathrm{H}}$ is the Hermitian transpose. Solving (3), the optimal weight vector is given as:

$$
\boldsymbol{w}_{\mathrm{SMI}}=\alpha \hat{\boldsymbol{R}}^{-1} \boldsymbol{a}_{s}
$$

where $\alpha=1 / \boldsymbol{a}_{s}^{H} \hat{\boldsymbol{R}}^{-1} \boldsymbol{a}_{s}$.

\section{IMPROVED MOdEL OF ARRAY STEERING VECTOR}

Affected by each antenna's radiation characteristics (e.g., directivity, gain), there are always mismatches between the ideal steering vector $\boldsymbol{a}$ and the actual one. In this paper, inspired by AP method, we use an improved array steering vector $\tilde{\boldsymbol{a}}$, by considering array aperture's radiation property [6]:

$$
\begin{aligned}
\tilde{\boldsymbol{a}}(f, \theta, \varphi, \boldsymbol{r}) & =\left[g_{1}(f, \theta, \varphi) e^{j k \boldsymbol{r}_{1}}, \cdots, g_{M}(f, \theta, \varphi) e^{j k \boldsymbol{r}_{M}}\right]^{T} \\
& =\operatorname{diag}(\boldsymbol{g}(f, \theta, \varphi)) \boldsymbol{a}(f, \theta, \varphi, \boldsymbol{r})
\end{aligned}
$$

where $\boldsymbol{g}(f, \theta, \varphi)=\left[g_{1}(f, \theta, \varphi), g_{2}(f, \theta, \varphi), \cdots, g_{M}(f, \theta, \varphi)\right]^{T}$, and $g_{i}(f, \theta, \varphi)$ is the known active gain response of the $i$ th antenna, which reflects array aperture's radiation abilities. $\boldsymbol{g}(f, \theta, \varphi)$ can be obtained and stored during the designing process of antennas by electromagnetic simulation software or actual measurements.

Although in practice, array's radiation performance can be affected by array mismatches such as the fluctuation of array parameters during design, processing, measuring, assembling and application, this new steering vector model using AP is still a better representation of the real one in the uncertain environment. It is mainly because these mismatches between the designed and applied array generally cause little changes of antennas' basic radiation structures, therefore have negligible impact on array's electromagnetic characteristics, e.g., current distribution and boundary conditions. Hence, the radiation in the near field stays almost the same. In the far field, the overall shapes of radiation pattern and the directivity tend to be similar, although the value of gain has a high probability of being different to the initially designed one [9].

In addition, as the active patterns are approximately continues versus angle, i.e., unlikely to have a value jump within a certain range. Thus, we can infer that when facing DOA mismatches (in a tolerable range), the new model $\tilde{\boldsymbol{a}}$ is still more approximate to the real one than $\boldsymbol{a}$. To sum up, it can be mathematically express as:

$$
\|\boldsymbol{e}\|>\|\tilde{\boldsymbol{e}}\| \quad \boldsymbol{e}=\hat{\boldsymbol{a}}-\boldsymbol{a}, \tilde{\boldsymbol{e}}=\hat{\boldsymbol{a}}-\tilde{\boldsymbol{a}}
$$

These inferences can also be validated by our own measurement results for practical arrays, and some of the representative results are given in Section V.

\section{Proposed APDL AlgorithM}

Referring to the DL method [1] and the AP method, we propose the APDL algorithm. By adding an extra loading item is added to the sample covariance matrix, its constrained problem can be express as:

$$
\min _{\boldsymbol{w}} \boldsymbol{w}^{H}(\hat{\boldsymbol{R}}+\xi \boldsymbol{I}) \boldsymbol{w} \text { s.t. } \quad \boldsymbol{w}^{H} \tilde{\boldsymbol{a}}_{s}=1
$$

Similar to the DL method, $\xi$ is the DL factor, and $\boldsymbol{I}$ denotes the identity matrix. Particularly, the ideal signal steering vector $\boldsymbol{a}_{s}$ in traditional DL method is modified as $\tilde{\boldsymbol{a}}_{s}$ in our method. Then, the weight vector of APDL can be written as:

$$
\boldsymbol{w}_{A P D L}=\alpha_{A P D L}(\xi \boldsymbol{I}+\hat{\boldsymbol{R}})^{-1} \tilde{\boldsymbol{a}}_{s}
$$

where $\alpha_{A P D L}=1 / \tilde{\boldsymbol{a}}_{s}^{H}(\xi \boldsymbol{I}+\hat{\boldsymbol{R}})^{-1} \tilde{\boldsymbol{a}}_{s}$. It has been proved that the loading item can reduce the impact of the array mismatches by reducing the interference of the small eigenvalues [4]. Usually, the DL factor is chosen in a more ad hoc way, typically $10 \sigma^{2}$. Here, $\sigma^{2}$ is the noise power in a single sensor.

Comparing with the AP method, the APDL method is more robust facing the performance degradation caused by the imprecise prestored data, as the effectiveness of the DL factor had been comprehensively discussed [3].

The APDL beamforming algorithm has a comparable computational cost of the DL method whose complexity is $O\left(M^{3}\right)$ per iteration. Although the APDL method needs to extract the prestored active gain data of the specific DOA, this procedure is barely time-consuming.

\section{Simulation Resulets}

We can also improve our experimental methods through the AP idea. Rather than hypothesize the array mismatches as statistical random errors, we can simulate the practical signal and interference with the electromagnetic data, and then simulate the narrow signal received by the array as:

$$
\hat{\boldsymbol{x}}(k)=\widehat{\boldsymbol{s}}(k)+\hat{\boldsymbol{i}}(k)+\boldsymbol{n}(k)=s(k) \hat{\boldsymbol{a}}_{s}+i(k) \hat{\boldsymbol{a}}_{i}+\boldsymbol{n}(k)
$$

where $\hat{\boldsymbol{x}}(k)$ is the changing input signals affected by the varying $\hat{\boldsymbol{a}}_{s}$ and $\hat{\boldsymbol{a}}_{i}$, due to the array's fluctuating response caused by mismatches, which reflects the changing environment. Correspondingly, the sample covariance matrix is:

$$
\hat{\hat{\boldsymbol{R}}}=\frac{1}{K} \sum_{i=1}^{K} \widehat{\boldsymbol{x}}(i) \hat{\boldsymbol{x}}^{H}(i)
$$

In this way, we create an experimental environment which is closer to the practical beamforming systems' working environment.

As shown in Fig. 1, a practical 4-element uniform circular microstrip array is used as a standard model in our experiments. The array works for an anti-interference system of BeiDou navigation. In this paper, the electromagnetic software HFSS is used for all antenna simulations. Fig. 2 shows the active patterns of each element. Obviously, the patterns are significantly different to the ideal omnidirectional models.

Assuming that there are some mismatches between the designed and the practical array, we simulate these different situations by HFSS. The corresponding AP of each mismatch is 


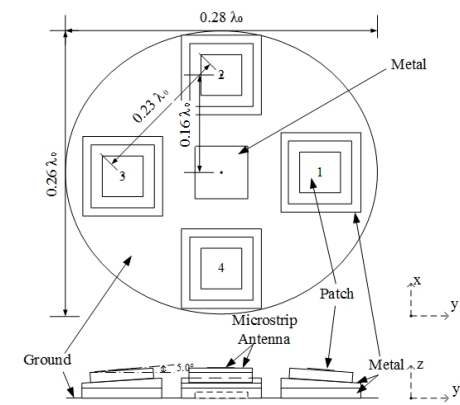

Fig. 1. A miniature circular microstrip array. Every right-handed circular polarized element's center frequency is $f_{0}=1.268 \mathrm{GHz}$. The dielectric substrate of each element has RDP of $\varepsilon_{r}=20$ and LT of $\tan \delta=0.004$. The metal working platform is elliptical, with a $2 a=0.281 \lambda=134 \mathrm{~mm}$ major axis and a $2 b=0.256 \lambda=121 \mathrm{~mm}$ minor axis. The array's radius is $r=0.16 \lambda=38 \mathrm{~mm}$ and the interelement spacing is $d=0.23 \lambda=54 \mathrm{~mm}$. Each antenna is set at an inclined angle of $\theta=5^{\circ}$ on the workbench.
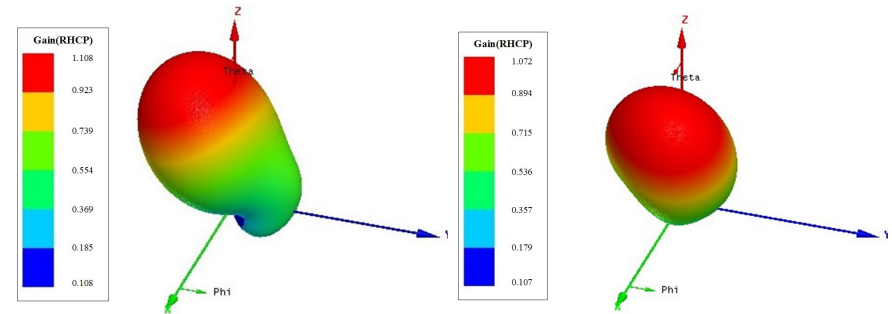

(a). The active pattern of element 1 .

(b). The active pattern of element 2 .
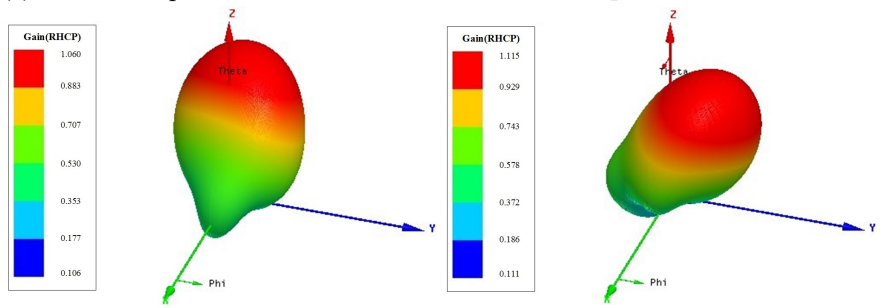

(d). The active pattern of element $4 . \quad$ (c). The active pattern of element 3.

Fig. 2. The active patterns of each element.

then abstracted and prestored. Three kinds of common engineering errors are studied: element's position mismatches (situation a), size errors of the metal working platform (situation b), and dielectric parameter errors of elements' substrate relative dielectric permittivity (RDP) (situation c) and loss tangent (LT) (situation d). Although rarely individually discussed previously in the adaptive beamforming algorithms, these mismatches are almost inevitable and will influence the whole array's radiation performance, and consequently cause beamformers' performance deterioration. The signal steering vector $\tilde{\boldsymbol{a}}_{s}$ used in AP and APDL method is calculated with the data extracted from the standard model, which is shown in Fig. 1. Meanwhile, $\hat{\boldsymbol{a}}_{s}, \hat{\boldsymbol{a}}_{i}$ and $\hat{\hat{\boldsymbol{R}}}$ that reflect the varying electromagnetic environment are changing according to different prestored data. In this way, we are able to simulate the performances of the APDL method and other typical beamformers when there are various mismatches. With the data of $\tilde{\boldsymbol{a}}_{s}$ and $\hat{\boldsymbol{a}}_{s}$, Fig. 3. illustrates $\|\boldsymbol{e}\|$ and $\|\tilde{\boldsymbol{e}}\|$ in (6) under different kinds of mismatches. Results are in accordance with (6).

In all simulations, the signal and interference source are assumed to have plane wavefronts, and DOAs are equal to $\left(\theta_{s}, \varphi_{s}\right)=\left(70^{\circ}, 6^{\circ}\right)$ and $\left(\theta_{i}, \varphi_{i}\right)=\left(1^{\circ}, 90^{\circ}\right)$ respectively. Table I compares element 1's gain versus angle around the signal's DOA. The results are in accordance with our inference in Section IV that the numerical value of gain tend to have a negligible change as long as the error of DOA is not serious.

In all experiments, 200 simulation runs are used to obtain each simulated point, the signal-to-noise-ratio(SNR) and interference-to-noise-ratio(INR) in a single sensor is equal to $25 \mathrm{~dB}$ and $30 \mathrm{~dB}$ respectively. Signals are always present in the training data cell (training data size $\mathrm{N}=100$ ). Additive noise in the array is modeled as spatially and temporally independent complex Gaussian noise with zero mean and unit variance. Five typical algorithms' mean output SINR are tested under mismatch situations: the SMI beamformer, the LSMI method [1], the WCRB [2] method using SeDuMi convex optimization MATLAB tool box, the AP beamformer [7], and the APDL method. The optimal SINR is also shown in the figures.

Fig. 4 presents the beamformers' performance towards element's position mismatches (element 1 is moved along the $y$ axis from $-2 \mathrm{~mm}$ to $2 \mathrm{~mm}, 1 \mathrm{~mm}$ per time).

As the working platform can cause the change of edge scattering and influence the electromagnetic radiation to the sensitive miniature array, in Fig. 5, we study how size errors of the metal working platform influence the beamformers, by setting the length of the elliptical metal platform's major axis from $132 \mathrm{~mm}$ to $136 \mathrm{~mm}$.

TABLE I

ELEMENT 1'S GAIN VERSUS ANGLE OF $\theta_{s}=70^{\circ}$

\begin{tabular}{|c|c|c|c|c|c|c|c|}
\hline Angle & $0^{\circ}$ & $2^{\circ}$ & $4^{\circ}$ & $6^{\circ}$ & $8^{\circ}$ & $10^{\circ}$ & $12^{\circ}$ \\
\hline Gain & 0.356 & 0.350 & 0.344 & 0.339 & 0.334 & 0.330 & 0.325 \\
\hline
\end{tabular}

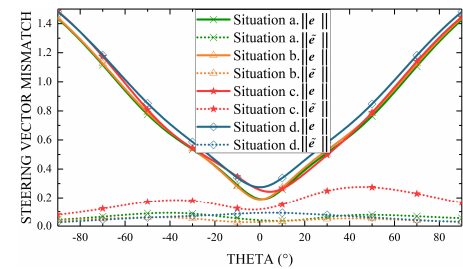

Fig. 3. Steering vector mismatches (on $\varphi=0^{\circ}$ plane) under different situations: a. Element 1 's position mismatch $(+2 \mathrm{~mm}$ along axis $\mathrm{y})$; b. Working platform mismatch ( $2 a=136 \mathrm{~mm})$; c. Element 1's RDP mismatch ( $\varepsilon_{r}=20.3$ ); d. Element 1's LT mismatch ( $\tan \delta=0.005$ ).

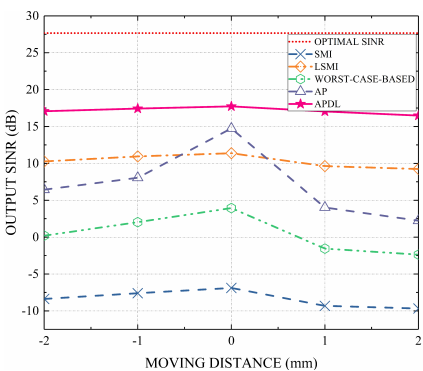

Fig. 4. Output SINR versus element 1 's position.

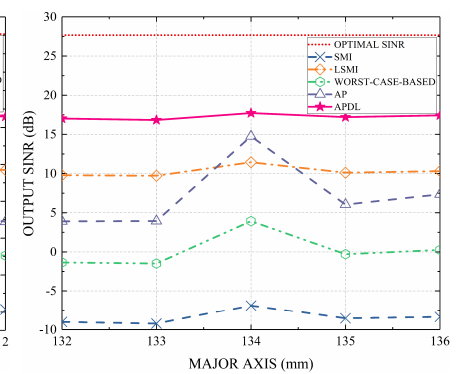

Fig. 5. Output SINR versus the length of the elliptical platform's major axis. 
TABLE II

The Active Gain of Designed ArRay $\left(G_{d}\right)$ AND the FABricAted ARray $\left(G_{f}\right)$ FROM THE DOA OF SIGNAL AND INTERFERENCE.

\begin{tabular}{|c|c|c|c|c|}
\hline \multirow{2}{*}{$\begin{array}{c}\text { Element's } \\
\text { number }\end{array}$} & \multicolumn{2}{|c|}{$G_{d}(\mathrm{~dB})$} & \multicolumn{2}{c|}{$G_{f}(\mathrm{~dB})$} \\
\cline { 2 - 5 } & $\theta_{s}=70^{\circ}, \varphi_{s}=6^{\circ}$ & $\theta_{i}=3^{\circ}, \varphi_{i}=90^{\circ}$ & $\theta_{s}=70^{\circ}, \varphi_{s}=6^{\circ}$ & $\theta_{i}=3^{\circ}, \varphi_{i}=90^{\circ}$ \\
\hline 1 & -4.91 & -0.40 & -3.03 & -0.48 \\
\hline 2 & -2.84 & -0.50 & -2.53 & -0.54 \\
\hline 3 & -3.62 & -0.24 & -3.38 & -0.53 \\
\hline 4 & -3.56 & -0.50 & -0.81 & -0.12 \\
\hline
\end{tabular}

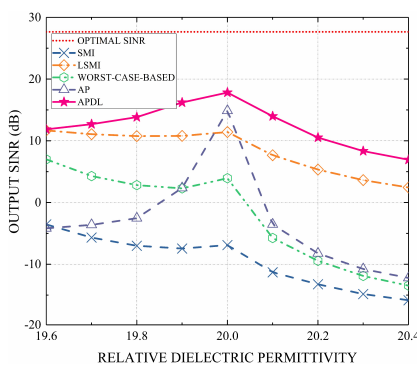

(a). Output SINR versus the element 1's substrate's RDP.

Fig. 6. The Dielectric Parameter Errors of Elements' Substrate

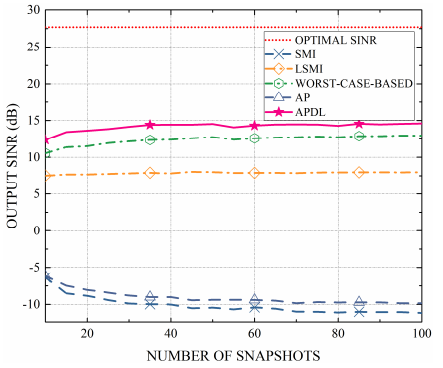

Fig. 7. Output SINR versus training data size for $\mathrm{SNR}=25 \mathrm{~dB}$.

We also study the situation when there are mismatches between the designed and the practical dielectric material, which may cause frequency deviation and radiation loss, and further influence antenna's radiation. Fig.6(a) shows the beamformers' output SINR when element 1's RDP varies from 19.6 to 20.4, while Fig. 6(b) demonstrates every elements' LT varies from 0.0005 to 0.005 . Value ranges in Fig. 6 are determined by the material's quality index and engineering experience.

Results shown in Fig. 2, Fig. 3 and Table $I$ are in accordance with our inferences in Section III-A. From Fig. 4 to Fig. 6, we can find that APDL has better performances than the other tested methods when facing various common engineering mismatches.

\section{MEASURED RESULTS}

The array mentioned in Section $\mathrm{V}$ was fabricated and debugged, and then measured in the microwave anechoic chamber. There are numerical differences of the AP between the designed and the practical array, as was expected. From the DOA of signal and interference, Table II compares the elements' simulated and measured active gain data. With the measured data, we assessed the aforementioned algorithms' performance using the same parameters (e.g., DOA and INR with those in simulation. Fig. 7 compares the mean SINR for the 6 methods with varying number of training snapshots. Fig. 8 shows how the mean output SINR changes with different SNRs.

These results based on the measurement demonstrate that the proposed APDL method perform satisfactorily in practice. The consistency between measurement and simulation results consolidate the effectiveness and robustness of the proposed APDL in dealing with array imperfectness. Measurement results are consistent with simulation results in Section IV-A. Those results prove that the previously gained array data can improve the beamformer's performance.

\section{CONCLUTION}

In this paper, we propose an improved adaptive beamforming algorithm, embedding arrays' electromagnetic characteristics in a robust beamformer. Mathematical analysis, computer simulation, and measurement results illustrate that the method performs well when facing possible array mismatches such as fluctuations between design, processing, measuring, assembling and application. The proposed method is particularly suitable for beamforming systems with small and compact arrays, where serious mutual coupling and environment scattering significantly influence antennas' radiation and consequentially the algorithm's performance.

\section{REFERENCES}

[1] H. Cox, R. Zeskind, and M. Owen, "Robust adaptive beamforming," IEEE Transactions on Acoustics, Speech, and Signal Processing, vol. 35, pp. 1365-1376, October 1987.

[2] S. A. Vorobyov, A. B. Gershman, and Z.-Q. Luo, "Robust adaptive beamforming using worst-case performance optimization: A solution to the signal mismatch problem," IEEE Transactions on Signal Processing, vol. 51, February 2003, pp. 313-324,.

[3] J. Li, and P. Stoica. Robust Adaptive Beamforming, vol. 88. John Wiley \& Sons, 2005.

[4] X. Gou, Z. Liu, and Y. Xu, "Fully automatic robust adaptive beamforming using the constant modulus feature," IET Signal Processing, vol. 8, 2014, pp. 823-830.

[5] X. Song, J. Wang, Y. Han, and Y. Xue "Robust adaptive beamforming under the quadratic inequality constraint," Wireless Communications, Networking and Mobile Computing, WiCom'09. 5th International Conference on. IEEE, 2009.

[6] B. $\mathrm{Ng}$, "Array shape self-calibration technique for direction finding problems," in IEE Proceedings H-Microwaves, Antennas and Propagation, vol. 139, IET, December 1992, pp. 521-525.

[7] R. Goossens and H. Rogier, "Optimal beam forming in the presence of mutual coupling," in Communications and Vehicular Technology, 2006 Symposium on. IEEE, 2006, pp. 13-18.

[8] D. F. Kelley and W. L. Stutzman, "Array antenna pattern modeling methods that include mutual coupling effects," IEEE Transactions on antennas and propagation, vol. 41, December 1993, pp. 1625-1632.

[9] R. John., "Antenna tolerance theory-A review," Proceedings of the IEEE, vol. 54, 1966, pp: 633-640. 